\title{
Wave Number Domain Analysis of Magnetometer Array Data
}

\author{
Xi-shuo Wang, J. C. SAmson, and D. I. Gough \\ Institute of Earth and Planetary Physics, Department of Physics, University of Alberta, \\ Edmonton, T6G 2J1, Canada
}

(Received May 22, 1986; Revised August 11, 1986)

\begin{abstract}
The paper makes a new contribution to the problem of separation of magnetovariation fields, observed over a limited area of the Earth's surface, into parts of external and internal origins. It is shown that there are important advantages in making such separations in the wavenumber domain, making use of the orthogonality of external and internal parts. The method uses a unified potential both for the adjustment of data to minimize their curl, and in the separation process. The technique is tested by combining the analytically calculated fields of line currents above and below the plane of "observation", separating them and comparing the separated field components with the known original fields. While the precision is limited by known effects of the limited area covered by the data, satisfactory correlation coefficients are obtained between separated and original fields. The new separation technique is finally applied to a magnetovariation event recorded in a recent array study in western Canada, and it is shown that a prominent anomaly is caused by internal currents.
\end{abstract}

\section{Introduction}

The interpretation of time-varying magnetic fields recorded at the Earth's surface involves the problem of separation of the observed field components into parts due to external and internal currents. Global data are usually expressed in spherical harmonics for this purpose (CHAPMAN and BARTELS, 1940) but for fields recorded over a limited area numerical integration is very often employed (VESTINE, 1941; PRICE and WILKINS, 1963; WEAVER, 1964; PORATH et al., 1970). Such numerical calculations are usually carried out in the space domain. The use of the wave number domain has two notable advantages: computing time is saved through the fast Fourier transform (FFT), and the results are ready for application of the induction equations in more easily soluble form. WIESE (1965) was the first to separate internal and external fields in the wave number domain, using a formulation which differed from this study in two respects. Working before the development of the FFT, Wiese considered only the real part of the Fourier transform, whereas the FFT provides the full complex values; and where the two horizontal components gave different estimates of parameters he used mean values. This is an ad hoc procedure without formal theoretical basis.

This paper presents a unified technique of potential field analysis in the 
wavenumber domain. The observed field is adjusted to be curl-free by calculating an optimum potential and separated into external and internal parts, if both exist. Magnetovariation data are here considered, but the technique is readily applicable to other potential fields.

2. Representation of a Potential Field in Cartesian Space and Wavenumber Domains

Consider a vectorial field $\boldsymbol{H}$ in a source-free region, here the observed magnetovariation field, derivable from a scalar potential $\Phi$ :

$$
\boldsymbol{H}(\boldsymbol{X})=-\nabla \Phi(\boldsymbol{X})
$$

where $\Phi(\boldsymbol{X})$ satisfies Laplace's equation:

$$
\nabla^{2} \Phi(\boldsymbol{X})=0
$$

The position vector $\boldsymbol{X}$ of a magnetometer has components $x$ (true north), $y$ (east) and $z$ (down). With $z=0$ in the plane of observation, $\boldsymbol{X}=(x, y, 0)$. The 2-dimensional Fourier transform of the potential is

$$
\begin{gathered}
\Phi(\boldsymbol{K}, z)=\iint \Phi(x, y, z) \exp \left[i K_{x} x+i K_{y} y\right] \mathrm{d} x \mathrm{~d} y \\
i=\sqrt{-1}
\end{gathered}
$$

Equation (2) then has the solution

$$
\Phi(\boldsymbol{K}, z)=B(\boldsymbol{K}) \exp [-v z]+A(\boldsymbol{K}) \exp [v z]
$$

where

$$
v=\sqrt{\left(K_{x}^{2}+K_{y}^{2}\right)}
$$

in the wavenumber domain, and $A$ and $B$ are terms representing internal and external sources respectively. Introducing vectors $\boldsymbol{K}_{\mathrm{a}}$ and $\boldsymbol{K}_{\mathrm{b}}$ :

$$
\boldsymbol{K}_{\mathrm{a}}=\exp (v z)\left[\begin{array}{c}
i K_{x} \\
i K_{y} \\
-v
\end{array}\right] \quad \boldsymbol{K}_{\mathrm{b}}=\exp (-v z)\left[\begin{array}{c}
i K_{x} \\
i K_{y} \\
v
\end{array}\right]
$$

the magnetic field vector in $\boldsymbol{K}$ space takes the compact form:

$$
\boldsymbol{H}(\boldsymbol{K})=A(\boldsymbol{K}) \boldsymbol{K}_{\mathrm{a}}+B(\boldsymbol{K}) \boldsymbol{K}_{\mathrm{b}}
$$

$\boldsymbol{K}_{\mathrm{a}}$ and $\boldsymbol{K}_{\mathrm{b}}$ are linearly independent vectors in a unitary space (see e.g. SAMSON, 1983) representing internal and external fields respectively. They are mutually orthogonal: 


$$
\boldsymbol{K}_{\mathrm{a}} \dagger \boldsymbol{K}_{\mathrm{b}}=0
$$

where $\uparrow$ denotes the Hermitean adjoint of a vector. Field components satisfying (6) are necessarily curl-free, as they are derivatives of a scalar potential: equation (6) is the wavenumber domain equivalent of (1).

3. Separation of the Field into External and Internal Parts

To separate the field into external and internal parts in the wavenumber domain, the sum-square error $Q$ is minimized at $z=0$ :

$$
Q=\sum_{k}\left[\boldsymbol{H}(\boldsymbol{K})-A(\boldsymbol{K}) \boldsymbol{K}_{\mathrm{a}}-B(\boldsymbol{K}) \boldsymbol{K}_{\mathrm{b}}\right]+\left[\boldsymbol{H}(\boldsymbol{K})-A(\boldsymbol{K}) \boldsymbol{K}_{\mathrm{a}}-B(\boldsymbol{K}) \boldsymbol{K}_{\mathrm{b}}\right] .
$$

Setting $\partial Q / \partial A^{*}(\boldsymbol{K})=\partial Q / \partial B^{*}(\boldsymbol{K})=0$, where ${ }^{*}$ denotes complex conjugate,

$$
\begin{array}{r}
\boldsymbol{K}_{\mathrm{a}} \dagger\left[\boldsymbol{H}(\boldsymbol{K})-A(\boldsymbol{K}) \boldsymbol{K}_{\mathrm{a}}-B(\boldsymbol{K}) \boldsymbol{K}_{\mathrm{b}}\right]=0 \\
\boldsymbol{K}_{\mathrm{b}} \dagger\left[\boldsymbol{H}(\boldsymbol{K})-A(\boldsymbol{K}) \boldsymbol{K}_{\mathrm{a}}-B(\boldsymbol{K}) \boldsymbol{K}_{\mathrm{b}}\right]=0,
\end{array}
$$

and as $\boldsymbol{K}_{\mathrm{a}} \dagger \boldsymbol{K}_{\mathrm{b}}=0$, we have:

$$
\begin{aligned}
& A(\boldsymbol{K})=\frac{\boldsymbol{K}_{\mathrm{a}} \dagger \boldsymbol{H}(\boldsymbol{K})}{\boldsymbol{K}_{\mathrm{a}} \dagger \boldsymbol{K}_{\mathrm{a}}} \\
& B(\boldsymbol{K})=\frac{\boldsymbol{K}_{\mathrm{b}} \dagger \boldsymbol{H}(\boldsymbol{K})}{\boldsymbol{K}_{\mathrm{b}} \dagger \boldsymbol{K}_{\mathrm{b}}} .
\end{aligned}
$$

Explicity in terms of magnetic field components:

$$
\begin{aligned}
& A(\boldsymbol{K})=-\frac{1}{2 v}\left[\frac{i K_{x} H_{x}(\boldsymbol{K})}{v}+\frac{i K_{y} H_{y}(\boldsymbol{K})}{v}+H_{z}(\boldsymbol{K})\right] \\
& B(\boldsymbol{K})=-\frac{1}{2 v}\left[\frac{i K_{x} H_{x}(\boldsymbol{K})}{v}+\frac{i K_{y} H_{y}(\boldsymbol{K})}{v}-H_{z}(\boldsymbol{K})\right] .
\end{aligned}
$$

The inverse Fourier transforms of $A(\boldsymbol{K})$ and $B(\boldsymbol{K})$ give the potentials in the space domain for internal and external fields respectively. From Parseval's theorem, the potential spectra defined above will give, within their resolution, the curl-free magnetic field closest to the original data.

4. Curl-Free Adjustment of the Field

If the field is entirely external or internal then all the components can be adjusted to make the field curl-free. Thus for an entirely external field, only $B(\boldsymbol{K})$ is finite in (6) 
and substitution of (12a) and (5) into (6) will adjust observed field components $H_{x}(K)$ etc. into $H_{x}$ etc. as:

$$
\begin{aligned}
& H_{x}=\frac{1}{2 v}\left[\frac{K_{x}^{2} H_{x}(\boldsymbol{K})}{v}+\frac{K_{x} K_{y} H_{y}(\boldsymbol{K})}{v}+i K_{x} H_{z}(\boldsymbol{K})\right] \\
& H_{y}=\frac{1}{2 v}\left[\frac{K_{y}^{2} H_{y}(\boldsymbol{K})}{v}+\frac{K_{y} K_{x} H_{x}(\boldsymbol{K})}{v}+i K_{y} H_{z}(\boldsymbol{K})\right] \\
& H_{z}=\frac{1}{2 v}\left[-i K_{x} H_{x}(\boldsymbol{K})-i K_{y} H_{y}+v H_{z}(\boldsymbol{K})\right] .
\end{aligned}
$$

In (6a) all three components are adjusted. This is possible because of the known source. With only external or only internal currents, so that either $A(\boldsymbol{K})$ or $B(\boldsymbol{K})$ is neglected, (4) gives the field variation with elevation. This makes possible the prediction of vertical derivatives of the field components required for complete curl-free adjustment.

In the presence of both external and internal sources, substitutions from (11) and (12) in (6) give the curl-free adjustment equations:

$$
\begin{aligned}
& \boldsymbol{H}_{x}=\left[K_{x}^{2} H_{x}(\boldsymbol{K})+K_{x} K_{y} H_{y}(\boldsymbol{K})\right] / v^{2} \\
& \boldsymbol{H}_{y}=\left[K_{y}^{2} H_{y}(\boldsymbol{K})+K_{y} K_{x} H_{x}(\boldsymbol{K})\right] / v^{2} \\
& \boldsymbol{H}_{z}=H_{z} \quad \text { (not adjustable) }
\end{aligned}
$$

Here only the horizontal components are adjusted because with both external and internal sources, the vertical gradient of the field cannot be predicted before separation.

Curl-free adjustment of the field before separation ensures that the gradients of the two horizontal components are consistent with one another. If the adjusted field is highly correlated with the original data, separation can proceed.

Singularities arise in (6a) and (6b) for uniform fields $(v=0)$. In practice such fields are inseparable, as are certain partially uniform components. In the $H_{x}\left(H_{y}\right)$ field a component uniform in the $x(y)$ direction having zero $K_{x}\left(K_{y}\right)$ is dropped from the observation and is neither adjustable nor separable. In (6a), for instance, spectrum components of all three field components having zero $K_{x}$ do not contribute to the adjusted field $H_{x}$. In practice the limitation is to fields of spatial wavelengths comparable to the array dimensions, or less. Similar limitations arise in separation in the space domain (OLDENBURG, 1969; PORATH et al., 1970).

A quantitative measure of the spatial similarity of two-dimensional complex fields $x$ and $y$, specified in discrete values $x(i, j), y(i, j)$ at grid points, is the correlation coefficient

$$
C=\lambda_{x y} / \sqrt{\left(\lambda_{x x} \lambda_{y y}\right)}
$$


where

$$
\begin{aligned}
& \lambda_{x y}=\sum_{i} \sum_{j}[x(i, j)-X][y(i, j)-Y]^{*} \\
& \lambda_{x x}=\sum_{i} \sum_{j}[x(i, j)-X][x(i, j)-X]^{*} \\
& \lambda_{y y}=\sum_{i} \sum_{j}[y(i, j)-Y][y(i, j)-Y]^{*}
\end{aligned}
$$

in which $X, Y$ are the averages of the two fields over the area. Correlation coefficients $C$ will be used to compare analytically calculated maps of test field components with corresponding curl-free adjusted maps; and with separated components of internal or external currents. It is important to note the meaning of a high value of $C$, approaching unity. $C$ is large for two mapped variables $x$ and $y$ if these have high spatial correlation of maxima and minima. However, $x$ and $y$ need not be equal. Thus $C=1.0$ if $y=a x+b$ with $a$ real and positive. $C$ is a suitable measure of the spatial similarity between an original, known field and a separated one. In the analyses of the data (to follow later) we shall use the Fourier coefficients of the time series in place of $x$ and $y$ in (13) and consequently $C$ will be complex. Imaginary parts of $C$ represent any resemblance of the real part of one field to the imaginary part of the other.

\section{Applications to Synthetic Data}

Tests have been made with synthetic and field data. Two synthetic models consist of line currents above and below the $(x, y)$ plane of observation. The vector magnetic field is calculated analytically at $15 \times 15$ grid points at $20 \mathrm{~km}$ spacing in $x$ and $y$. The curl-free adjustment is then applied, only to the horizontal field as both external and internal fields are present, and the fields are separated. To reduce distortion near the edges the data array is expanded symmetrically north-south and east-west before Fourier transformation of the magnetic field components (KANASEWICH, 1981, p.58). As uniform fields are inseparable, the average value for the expanded array is subtracted.

The first model consists of horizontal line currents in orthogonal azimuths, one of $90 \mathrm{kA}$ situated $90 \mathrm{~km}$ above the $(x, y)$ plane and the other of $40 \mathrm{kA}$ situated $80 \mathrm{~km}$ below it. The calculated fields, with straight contours, are shown in Figs. 1(a) and 2(a), and the combined original fields are shown in Fig. 3(a). The curl-free horizontal fields are shown in Fig. 3(b). Comparison of Fig. 3(a) and 3(b) indicates that the curl-free adjustment has not much altered the patterns of $X$ and $Y$ contours, and this is confirmed by correlation coefficients between curl-free and original fields of 0.857 for $X$ and 0.957 for $Y$ over the 225 grid points. As the calculated fields are in fact curl-free, the minor changes introduced in the curl-free adjustment must arise from the finite area of separation and the finite grid spacing.

The separated fields are shown in Figs. 1(b) and 2(b). The central parts of the maps preserve the parallel straight contours of the original fields. As expected, edge effects distort the margins. The correlation coefficients between the calculated and 
134

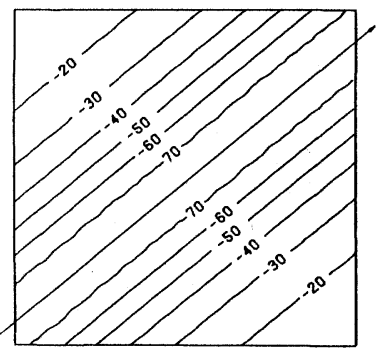

$\mathbf{x}$

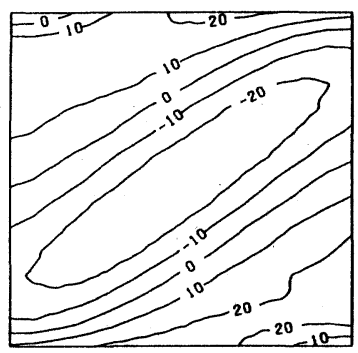

$\mathrm{X}$-s. WANG et al.

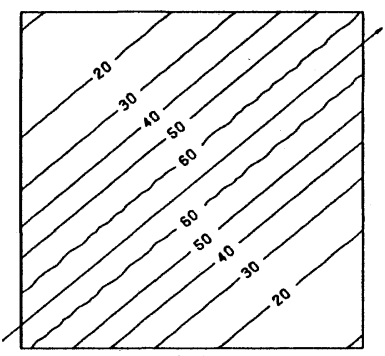

(a)

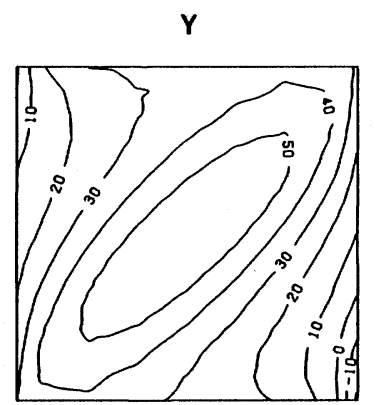

(b)

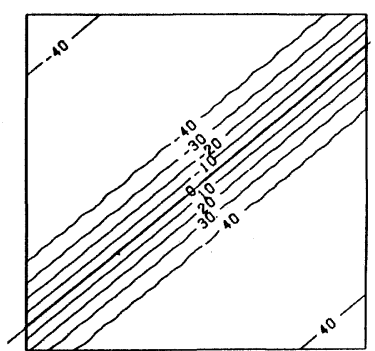

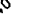

Fig. 1. (a) Calculated field components for a horizontal internal line current of $40 \mathrm{kA}$ flowing $80 \mathrm{~km}$ below the $(X-Y)$ plane. (b) Separated field components for the same current. Unit: $\mathrm{nT}$.

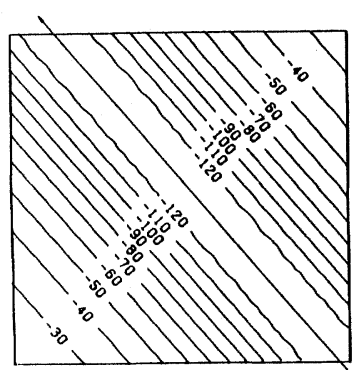

$\mathbf{X}$

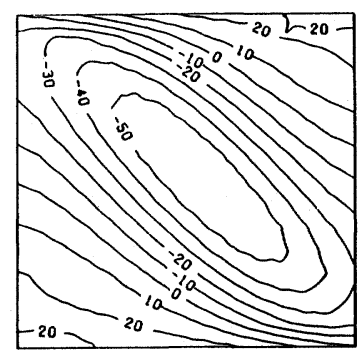

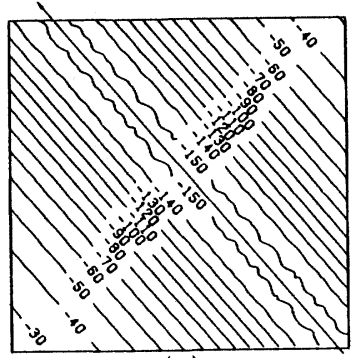

(a)

$\mathbf{Y}$

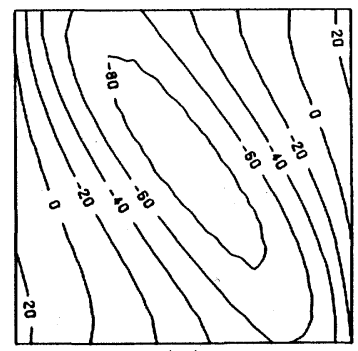

(b)

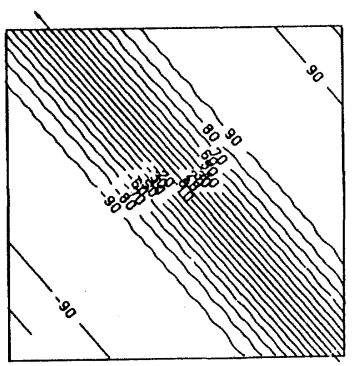

Z

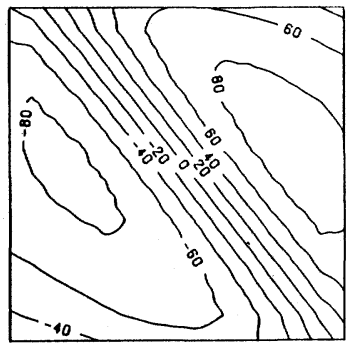

Fig. 2. (a) Calculated field components for a horizontal external line current of $90 \mathrm{kA}$ flowing $90 \mathrm{~km}$ above the $(X-Y)$ plane. (b) Separated field components for the same current. Unit: $\mathrm{nT}$. 


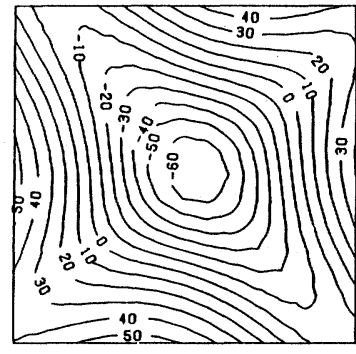

$\mathbf{x}$

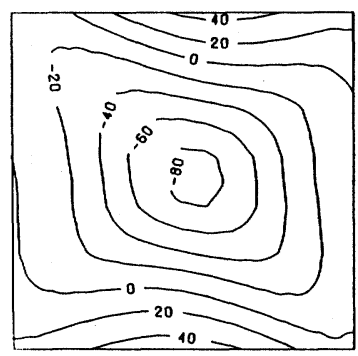

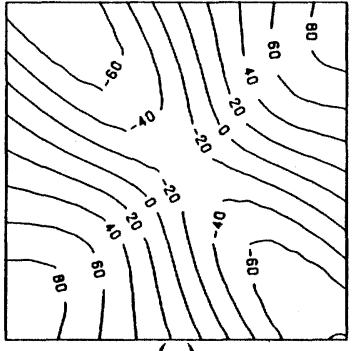

(a)

Y

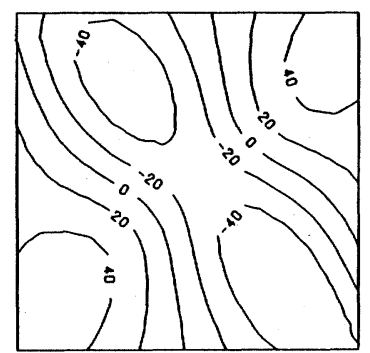

(b)

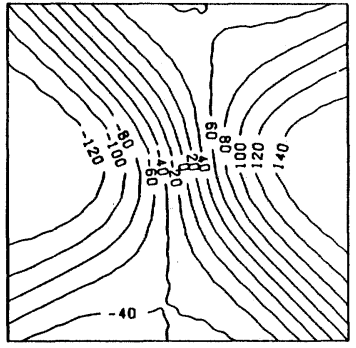

$\mathbf{Z}$

Fig. 3. (a) Components of the combined original field of external and internal line currents (see Figs. 1 and 2). (b) Curl-free horizontal components of the combined fields. Unit: nT.

separated internal fields are 0.826 for $X, 0.819$ for $Y$ and 0.849 for $Z$. For the external components these are $0.854,0.888$ and 0.984 . This model, with its two-dimensional currents and fields, necessarily contains large totally and partially uniform, and so inseparable parts. It provides a severe test of the method, with satisfactory results.

For the second synthetic model the external and internal fields are produced by two line currents, each in three segments, none horizontal. The external current is 90 $\mathrm{kA}$ and the internal $40 \mathrm{kA}$. The end segments of each flow from and to infinity: those of the internal current make angles of 75 and $70^{\circ}$ with the downward $\mathrm{z}$ axis and connect with the middle segment at depths $z=80$ and $75 \mathrm{~km}$. The from (to) infinity segment of the external current makes angle $110\left(120^{\circ}\right)$ with the $z$ axis and connect with the middle segment at $z=-80(-90) \mathrm{km}$. These three-dimensional currents have no large, inseparable parts and in consequence the separation is much more successful than that of the previous model. Figures 4(a) and 5(a) show the internal and external calculated fields, with horizontal projections of the currents, and Fig. 6(a) the total field. The curl-free horizontal fields of Fig. $6(\mathrm{~b})$ closely resemble the calculated total fields, with correlation coefficients 0.886 for $X$ and 0.981 for $Y$. The separated fields, shown in Figs. 4(b) and 5(b), match the model fields well: correlation coefficients for the calculated and separated internal and external fields are given in Table 1. 
136

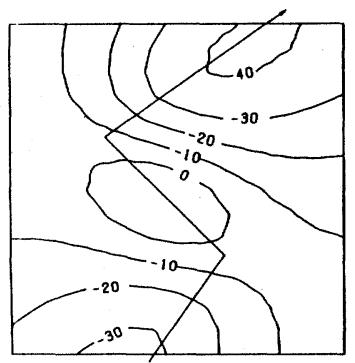

$x$

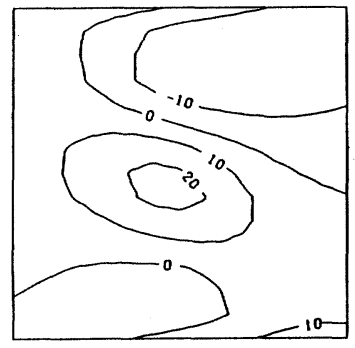

X-s. WANG et al.

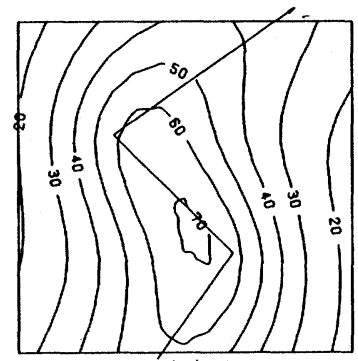

(a)

$\mathbf{Y}$

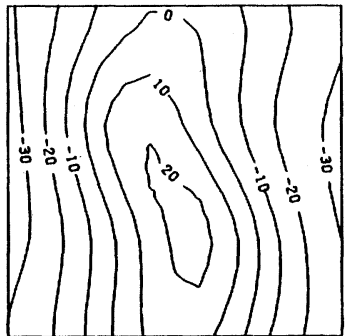

(b)

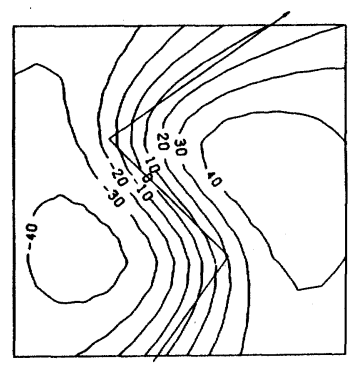

Z

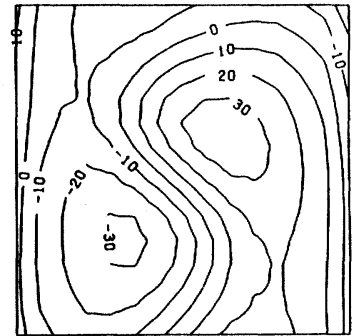

Fig. 4. (a) Calculated field components for a three-segment $40 \mathrm{kA}$ internal line current, and horizontal projection of the current. (b) Separated field components for the same current. Unit: nT.

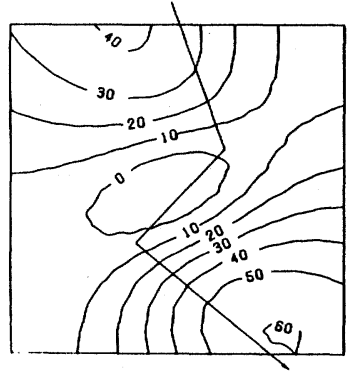

$x$

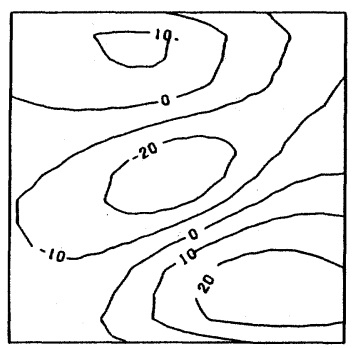

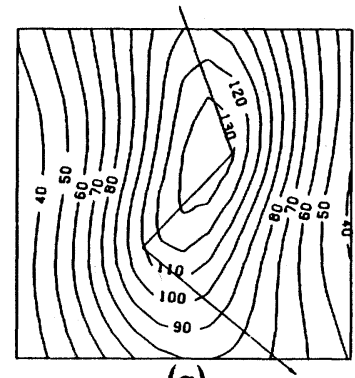

(a)

$Y$

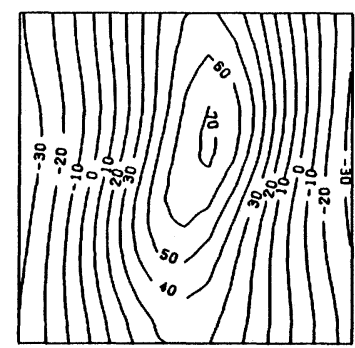

(b)

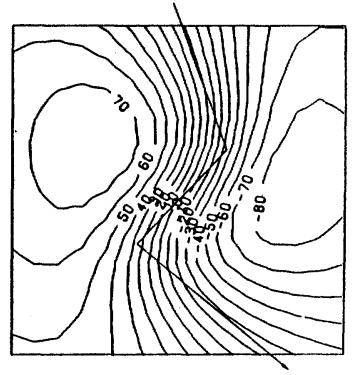

$\mathbf{Z}$

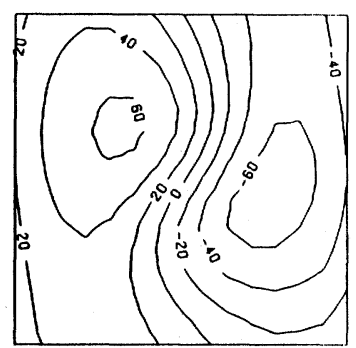

Fig. 5. (a) Calculated field components for a three-segment $90 \mathrm{kA}$ external line current, and horizontal projection of the current. (b) Separated field components for the same current. Unit: nT. 


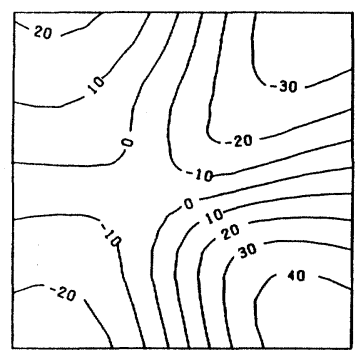

$\mathbf{X}$

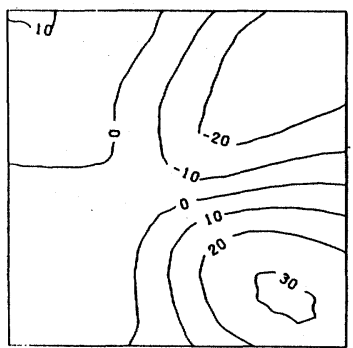

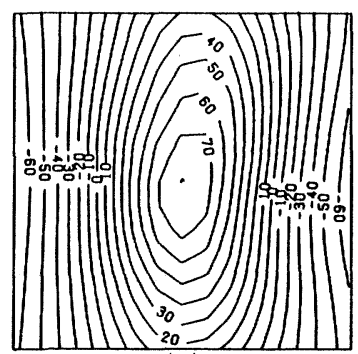

(a)

Y

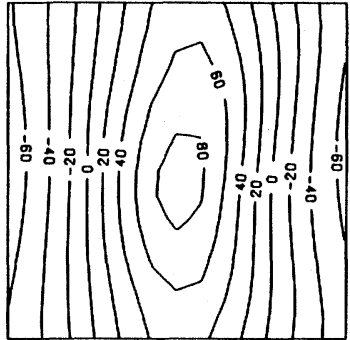

(b)

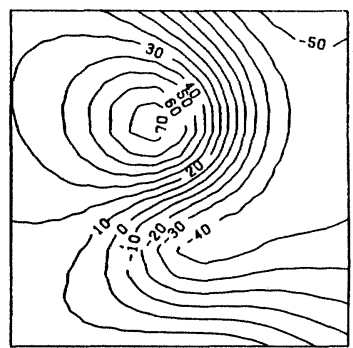

$\mathbf{Z}$

Fig. 6. (a) Components of the combined original field of three-segment external and internal line currents (see Figs. 4 and 5). (b) Curl-free horizontal components of the combined fields. Unit: nT.

Table 1. Correlation coefficients between original field components and those separated with and without data expansion.

\begin{tabular}{llcc}
\hline Field component & \multicolumn{2}{c}{$C$} \\
& & Expanded & Unexpanded \\
\hline Internal & $X$ & 0.929 & 0.833 \\
& $Y$ & 0.983 & 0.837 \\
& $Z$ & 0.749 & 0.620 \\
External & $X$ & 0.947 & 0.848 \\
& $Y$ & 0.988 & 0.960 \\
& $Z$ & 0.965 & 0.948 \\
\hline
\end{tabular}

\section{Edge Effects}

Separation of fields known over a limited area results in repetition of those fields to infinity along both $x$ and $y$ axes. If $A B$ represents the known fields in the space domain, the field separated is 
ABABAB $A B A B A B A B \ldots .$.

Distortions are produced, specially near the edges of the area of known field, arising mainly from the large discontinuities there. These edge effects can be reduced by expanding the known area to repeat the known fields in mirror image, as

\section{BAАВBA $A B$ BAABBA......}

to remove the edge discontinuities (KANASEWICH, 1981). The fields separated in Sections 5 and 7 have been expanded in this way.

An example of the improvement produced by the mirror-image expansion is provided in Fig. 7, which shows the internal parts of the magnetic field for the second synthetic data set, separated with and without expansion. The fields separated using expansion are shown also in Fig. 4, together with the analytically calculated fields of the internal current. Considerable distortions can be seen in the internal fields from the unexpanded separation of Fig. 7(b), notably near the north and south edges of the $X$ component map and the east and west edges of the $Y$ map. Table 1 shows the

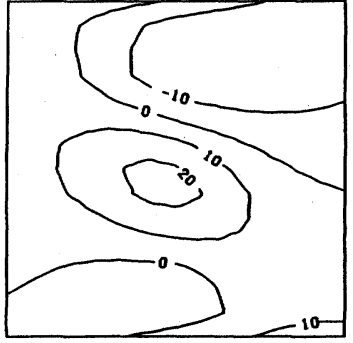

$\mathbf{X}$

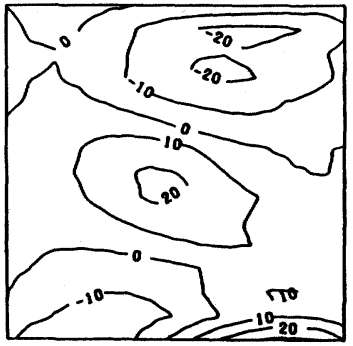

Fig. 7. Components of the field of the internal three-segment line current (see Fig. 4) separated (a) with mirror-image expansion of the area of known field, (b) without expansion. Figs. 4(b) and 7(a) are the same. Unit: $\mathrm{nT}$.

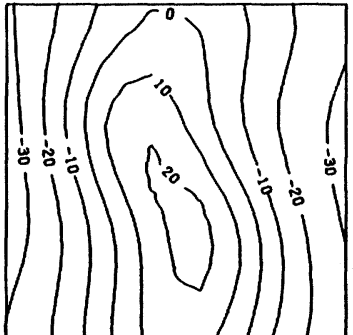

(a)

$Y$

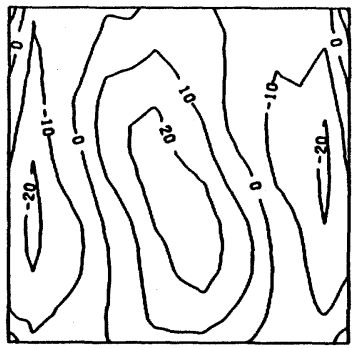

(b)

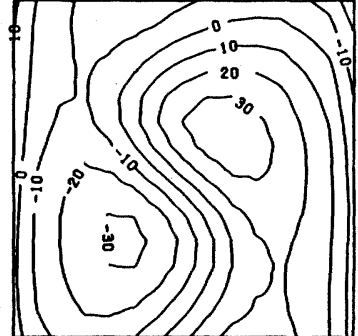

Z

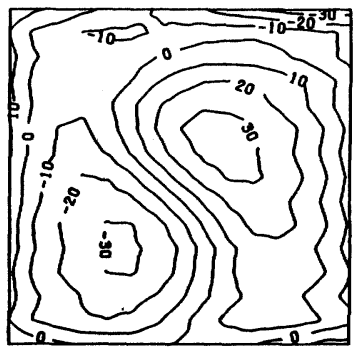



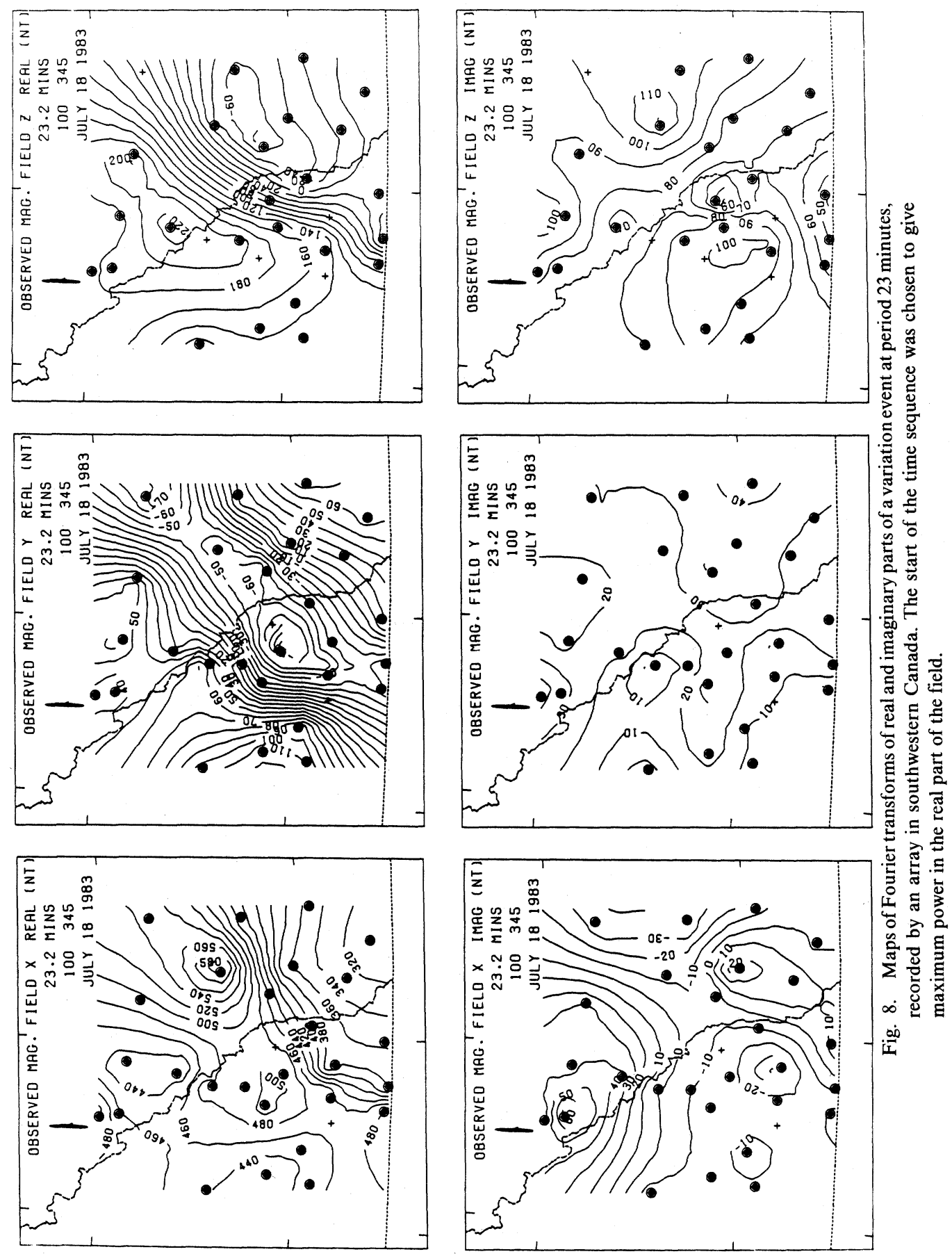
correlation coefficients between the original and the separated components, from expanded and unexpanded data. It is reassuring to note that the central part of each map is similar for separation of expanded and unexpanded fields.

\section{Application to Field Data}

A two-dimensional magnetovariation array was operated in 1983 over an area of $350 \times 350 \mathrm{~km}$ in southern Alberta and British Columbia, as part of a programme of study of conductive structures in western Canada. Its results will be published in full elsewhere. The separation technique here developed has been applied to a set of maps of Fourier transform coefficients at a period of 23.2 minutes, from a single magnetovariation event recorded at 27 stations (Fig. 8). The preparation and use of such maps has been described by GoUGH and INGHAM (1983). For the present separation, common phase maps must be used, showing "real" and "imaginary" fields relative to an arbitrary zero phase (PORATHet al., 1970). In Fig. 8 the zero phase has been chosen to maximize the real fields, so as to maximize power in one set of maps. In the three upper maps of Fig. 8 the fields of currents flowing NE-SW are immediately apparent.

Horizontal real components adjusted to be curl-free are shown in Fig. 9. The correlation coefficients over 225 grid points with the values of Fig. 8 are $0.846-0.047 i$ for the $X$ and $0.792+0.083 i$ for the $Y$ component. For the real maps the correlation is good. The smaller imaginary parts (not shown) show less significant correlation and are clearly more noise-contaminated.

In Fig. 10 the internal fields are larger and show more structure than external parts. Some contamination of the latter with internal fields is suggested by features in
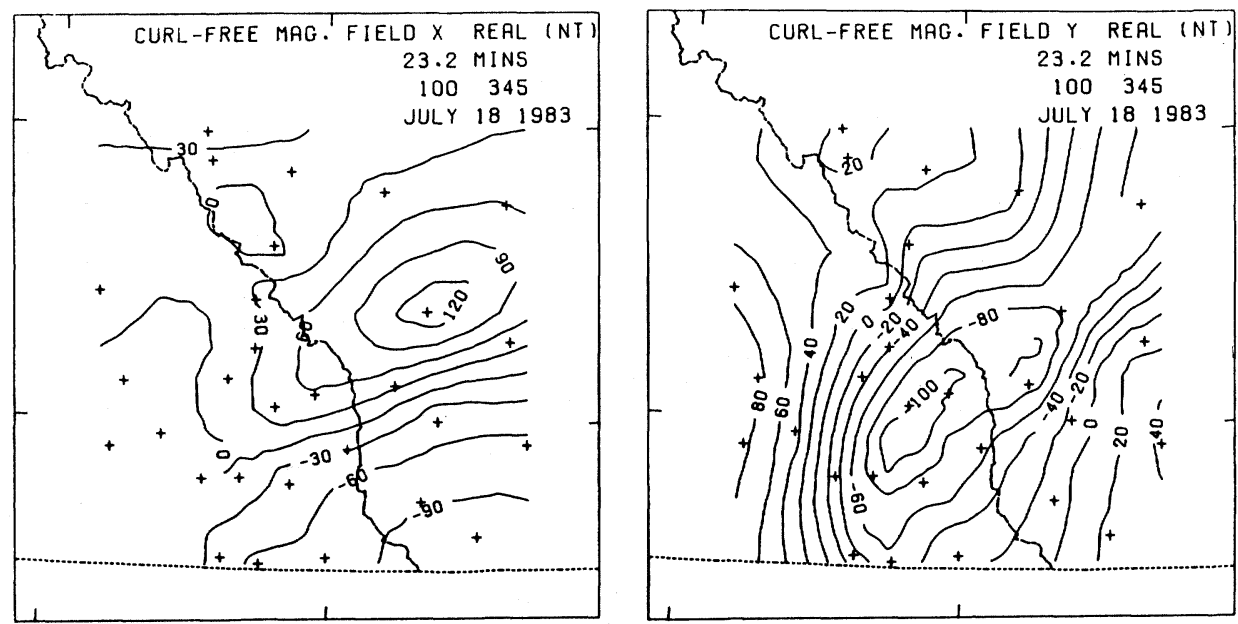

Fig. 9. Curl-free horizontal components of the real part of the magnetovariation event of Fig. 8 . 

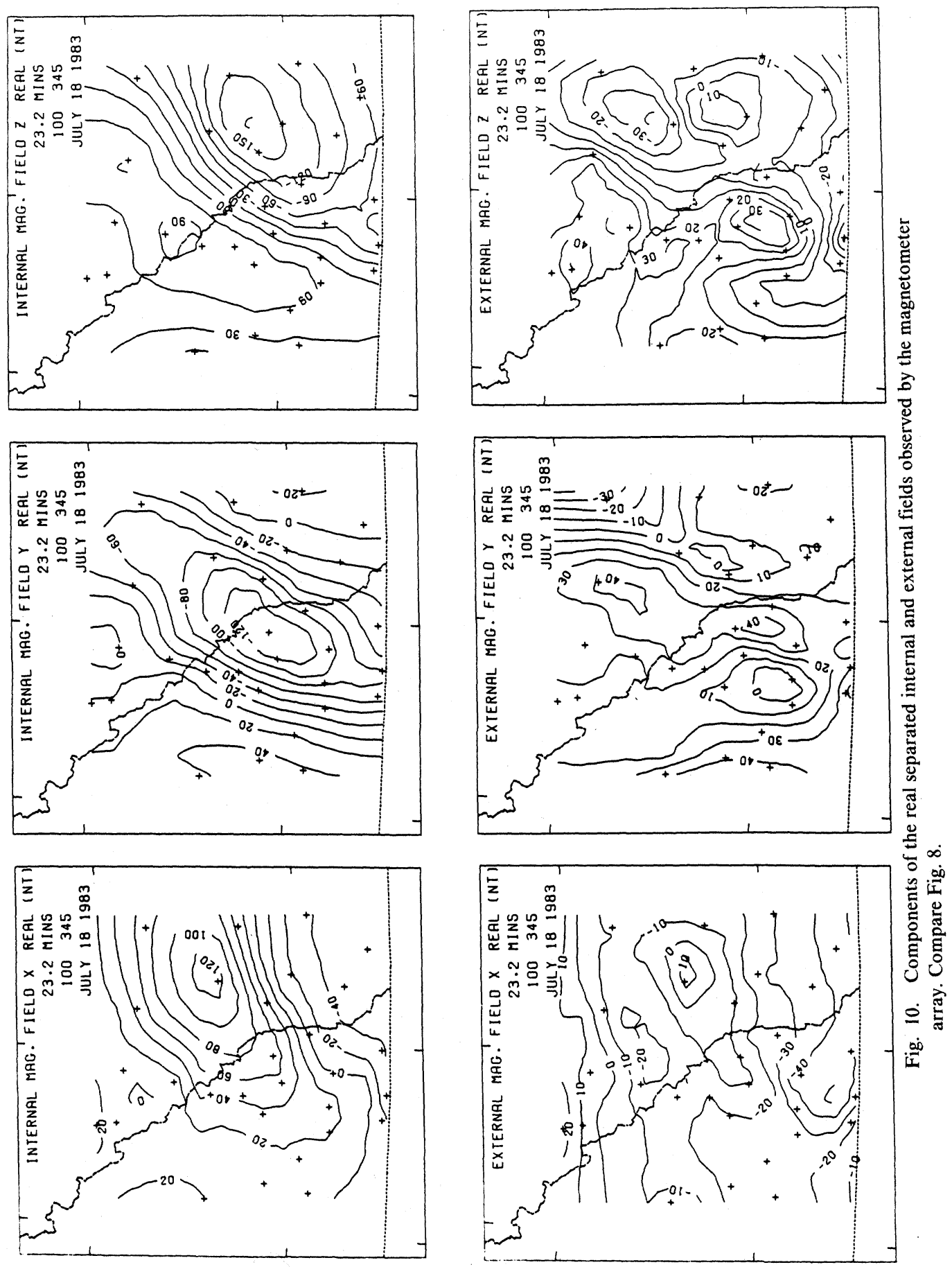
the external maps similar to the much larger anomalies in the internal maps. The limited size of the array and the edge effects probably produce some leakage of the internal fields into the external maps.

The maps of internal fields indicate a current curving from a north-northeast azimuth at the southern edge of the array to ENE at the eastern limit. The separation exercise supports the view that these currents are internal. The maps of external parts are contaminated with imperfectly separated internal fields and do not properly represent the fields of external currents.

For modelling of conductive structures one needs to estimate both external and internal fields, and to normalize the latter in terms of the former. Here the finite array, and the inseparability of fields of wavelengths larger than the array, presents a formidable problem. Any uniform, or very long wavelength, field cannot be separated. If the external field is mainly of this kind, it cannot be evaluated and the normalization is impossible. The problem has been discussed by OLDENBURG (1969), by PORATH et al. (1970) and by GOUGH and INGHAM(1983).

Despite its limitations, which have been noted, the separation technique given in this paper is effective for many magnetovariation fields, simple to use and computationally efficient. In many cases it will provide a clear indication that internal fields, external fields or both are present.

We thank Chen Guangming and K. Wilson for allowing us the use, in Section 7, of magnetovariation data to be reported in a paper of which they will be co-authors, and for much help with data processing. Dr. D. McA. McKirdy contributed helpful discussion. This research is supported by grants to D.I.G. from the Natural Science and Engineering Research Council of Canada.

\section{REFERENCES}

Chapman, S. and J. Bartels, Geomagnetism, 1049pp., Oxford University Press, 1940.

Gough, D. I. and M. R. InGHAM, Interpretation methods for magnetometer arrays, Rev. Geophys. Space Phys., 21, 4, 805-827, 1983.

KanaSewich, E. R., Time Sequence Analysis in Geophysics, 480pp., University of Alberta Press, 1981.

OldENBURG, D. W., Separation of magnetic substorm fields for mantle conductivity studies in the western United States, unpublished M. Sc. thesis, Univ. of Alberta, Edmonton, 1969.

Porath, H., D. W. Oldenburg, and D. I. Gough, Separation of magnetic variation fields and conductive structure in the western United States, Geophys. J. R. Astro. Soc., 19, 237-260, 1970.

Price, A. T. and G. A. Wilkins, New methods for the analysis of geomagnetic fields and their application to the Sq field of 1932-3, Philos. Trans. R. Soc. London, Ser. A, 256, 31-98, 1963.

SAmson, J. C., The spectral matrix, eigenvalues and principal components in the analysis of multichannel geophysical data, Annales Geophys., 1, 2, 115-119, 1983.

VeSTINE, E. H., On the analysis of surface magnetic fields by integrals, part I, Terr. Magn. Atmos. Electr., 46, 27-41, 1941.

WeAver, J. T., On the separation of local geomagnetic fields into external and internal parts, Zeits. Für Geophys., 30, 29-36, 1964.

WIESE, H., Geomagnetische Tiefentellurik, Deutsche Akad. Wiss. Berlin, Geomagn. Inst. Potsdam, Abh., 36, 1-146, 1965. 